\title{
Influence of the relative dimensions of the cavity on the conjugate convective heat transfer and on the shape of crystallization fronts in the method of Horizontal Directed Crystallization
}

\author{
Stepan A. Kislitsyn ${ }^{1,2, *}$ \\ ${ }^{1}$ Novosibirsk State Technical University, Novosibirsk, 630073, K. Marx Ave., 20, Russia \\ ${ }^{2}$ Kutateladze Institute of Thermophysics SB RAS, Novosibirsk, 630090, Ak. Lavrentiev Ave., 1, \\ Russia
}

\begin{abstract}
The process of water crystallization in rectangular cavities is numerically studied. The liquid being studied is water having an inverse temperature dependence of the density. Calculations were carried out with a suddenly cooled vertical wall of a rectangular cavity. The initial temperature of the melt is maintained on the opposite wall. The finite element method was used to solve a system of equations for the nonstationary free convection of a melt, taking into account the dependence of the density on temperature. The heat conduction equation in the solidified substance was also solved. The problems were solved in a conjugate formulation taking into account the release of the latent heat of crystallization at the liquid-solid interface.
\end{abstract}

\section{Introduction}

The method of Horizontal Directed Crystallization (HDC) is one of the popular methods for obtaining single crystals from melts [1]. As in other methods of directed crystallization, crystal growth occurs under conditions of conjugated convective heat transfer. In the HDC method, depending on the conditions at the upper boundary, the growth occurs in the regime of nonstationary thermogravitational or gravitational-capillary convection $[2,3]$ complicated by a phase transition. A simplified model of the technological process of HDC is the process of crystallization of the melt in a rectangular cavity with vertical walls heated up at different temperatures. The shape of crystallization fronts and the temperature field and the temperature gradients in the solidified material depend on the features of local heat transfer, on which the homogeneity of crystallographic characteristics of crystals is depended.

The use of water as a melt imitation fluid in the physical and numerical modeling of convective heat transfer is due to the inverse dependence of its density on temperature in the vicinity of $4^{\circ} \mathrm{C}$. Melt, for example, gallium and eutectic cadmium-mercury-tellurium and some other substances and materials have similar dependences. The inverse

\footnotetext{
*Corresponding author: 100pch@mail.ru
} 
dependence of the melt density on temperature near the crystallization temperature introduces significant features into the hydrodynamics of melts at the crystallization front and in the distributions of local heat fluxes. A consequence is the effect on the shape of the crystallization front.

\section{Formulation of the problem}

The problem was solved in a nonstationary two-dimensional conjugate formulation in Cartesian coordinates taking into account the latent heat of crystallization with different boundary conditions at the upper boundary. The problems were solved at different cavity sizes: $105 \times 210 \mathrm{~mm}^{2}, 105 \times 105 \mathrm{~mm}^{2}, 200 \times 50 \mathrm{~mm}^{2}, 200 \times 25 \mathrm{~mm}^{2}, 200 \times 12.5 \mathrm{~mm}^{2}$. At the initial time, the calculation area consists of a melt layer. After a sudden cooling of the right vertical wall below the freezing point, the crystallization process begins [4]. The initial temperature of the system is maintained on the left vertical wall. Convective heat transfer in a fluid is described by the dimensionless system of Navier-Stokes equations and energy in the Boussinesq approximation, written in terms of temperature, vorticity and stream function:

$$
\begin{gathered}
\frac{\rho_{f}(T)}{\rho_{f}}\left(\frac{\partial T}{\partial t}+u \frac{\partial T}{\partial x}+v \frac{\partial T}{\partial y}\right)=\frac{1}{P r}\left(\frac{\partial^{2} T}{\partial y^{2}}+\frac{\partial^{2} T}{\partial x^{2}}\right), \\
\frac{\rho_{f}(T)}{\rho_{f}}\left(\frac{\partial \omega}{\partial t}+u \frac{\partial \omega}{\partial x}+v \frac{\partial \omega}{\partial y}\right)=\left(\frac{\partial^{2} \omega}{\partial y^{2}}+\frac{\partial^{2} \omega}{\partial x^{2}}\right)+G r \frac{\partial T}{\partial x}, \\
\frac{\partial^{2} \psi}{\partial y^{2}}+\frac{\partial^{2} \psi}{\partial x^{2}}=-\omega, v=-\frac{\partial \psi}{\partial x}, u=\frac{\partial \psi}{\partial y}, G r=\frac{g \cdot \beta_{f}(T) \cdot H^{3} \Delta T}{v_{f}^{2}} .
\end{gathered}
$$

The equation of energy in the crystallization zone is modified taking into account the latent heat of crystallization:

$$
\left(1+\frac{R}{\mathrm{c}_{p f} \Delta T}\right)\left(\frac{\partial T}{\partial t}+u \frac{\partial T}{\partial x}+v \frac{\partial T}{\partial y}\right)=\frac{\lambda_{s}}{\lambda_{f} \cdot \operatorname{Pr}}\left(\frac{\partial^{2} T}{\partial y^{2}}+\frac{\partial^{2} T}{\partial x^{2}}\right) .
$$

Conductive heat transfer in a crystal is described by the equation of conduction:

$$
\frac{\rho_{s} c_{s}}{\rho_{f} c_{p f}} \frac{\partial T}{\partial t}+\frac{\lambda_{s}}{\lambda_{f} \cdot \operatorname{Pr}}\left(\frac{\partial^{2} T}{\partial x^{2}}+\frac{\partial^{2} T}{\partial y^{2}}\right)=0 .
$$

When solving the equations of motion, the temperature dependence of the coefficient of volumetric thermal expansion of water $\beta_{\mathrm{f}}(\mathrm{T})$ and its density $\rho_{\mathrm{f}}(\mathrm{T})$. To calculate the constant parameters, fixed values of the thermal properties of water and ice were used at $0^{\circ} \mathrm{C}$. Water properties: density $\rho_{\mathrm{f}}=999.839 \mathrm{~kg} / \mathrm{m}^{3}$; coefficient of kinematic viscosity $v_{\mathrm{f}}=1.793 \cdot 10^{-6}$ $\mathrm{m}^{2} / \mathrm{s}$; coefficient of dynamic viscosity $\mu_{\mathrm{f}}=1.792 \cdot 10^{-3} \mathrm{~Pa} \cdot \mathrm{s}$; coefficient of thermal conductivity $\lambda_{\mathrm{f}}=0.554 \mathrm{~W} /(\mathrm{m} \cdot \mathrm{K})$; specific heat $\mathrm{c}_{\mathrm{pf}}=4218 \mathrm{~J} /(\mathrm{kg} \cdot \mathrm{K}) ;$ latent crystallization heat $\mathrm{R}=333.7 \mathrm{~kJ} / \mathrm{kg}$. Properties of ice: $\rho_{\mathrm{s}}=916.2 \mathrm{~kg} / \mathrm{m}^{3} ; \lambda_{\mathrm{s}}=2.2 \mathrm{~W} /(\mathrm{m} \cdot \mathrm{K}) ; \mathrm{c}_{\mathrm{s}}=2050$ $\mathrm{J} /(\mathrm{kg} \cdot \mathrm{K})$.

At solid boundaries in the system and at the liquid-crystal interface, the conditions of adhesion: 


$$
\left.\psi\right|_{\Gamma}=0,\left.\omega\right|_{\Gamma}=\frac{\partial v}{\partial x}-\frac{\partial u}{\partial y} .
$$

The horizontal boundaries are adiabatic. The problem was solved in a dimensionless form, the height $(\mathrm{H})$ of the liquid layer was chosen as the scale of the geometric dimensions. For velocity and time, the scales $v_{\mathrm{f}} / \mathrm{H}$ and $\mathrm{H}^{2} / v_{\mathrm{f}}$ were used, respectively. The temperature scale is $\Delta \mathrm{T}=\mathrm{T}_{\mathrm{h}}-\mathrm{T}_{\mathrm{c}}$, where $\mathrm{T}_{\mathrm{h}}=+10^{\circ} \mathrm{C}$ and $\mathrm{T}_{\mathrm{c}}=-10^{\circ} \mathrm{C}$ is the temperatures on the hot and cold walls, respectively. The calculations were carried out by the finite element method using an adaptive triangular grid with the number of nodes from 35.000 to 65.000 . The triangular grid tracks the position of the crystallization front at each time step and thickens on both sides of it, and also to varying degrees to all boundaries of the computational domain.

\section{Numerical results}

This article presents numerical results obtained in cavities with different length and height relationships for a single temperature difference between vertical walls. For all geometries of the computational domain with the upper rigid boundary in Fig. 1-4 shows the positions of the crystallization front and the fields of the stream function isoline in steady-state stationary states.

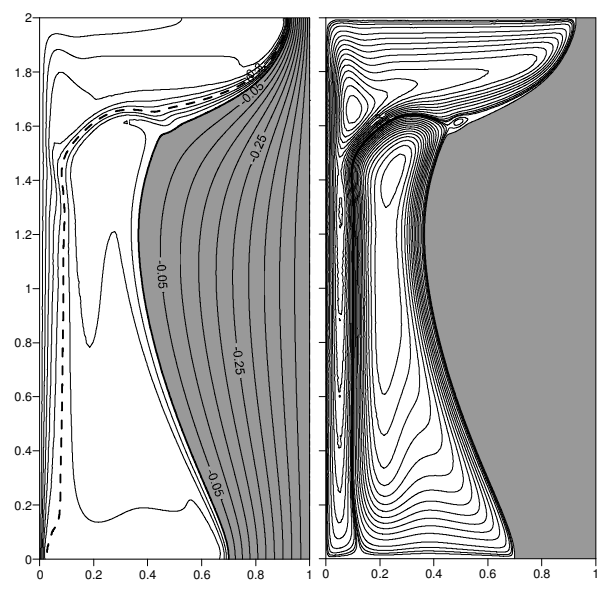

Fig. 1. Fields of isotherms and stream functions in steady state: height $=210 \mathrm{~mm}$, width $=105 \mathrm{~mm}$. Upper boundary with no-slip condition.

The inverse dependence of the density of water on temperature leads to features of the spatial form of the flow. The flow of heated liquid in high cavities (Fig. 1-3) reaches the ice layer in the upper part. Here the chilled water flows down. In the lower part of the crystallization front, a critical mass of water with a temperature in the range from 0 to + $4^{\circ} \mathrm{C}$ is accumulated. Here, a flow of liquid flows along the crystallization front from the bottom to the top. As a result, fluid circulation is observed clockwise in the upper part of the liquid layer. In the bottom part there is a cell with a liquid circulation counterclockwise. For all regimes where two convective cells are formed during the convective heat transfer, the maximum growth rate of the crystal is observed near the upper part of the convective cell with temperature $\leq 4^{\circ} \mathrm{C}$ (Fig. 1-4). The relative position of convective cells determines the type of curvature of the shape of the crystallization front. In the case where the height of the calculation domain is $12.5 \mathrm{~mm}$ and the rigid upper boundary (Fig. 4) 
allows the convective cell with a temperature $\leq 4^{\circ} \mathrm{C}$ to occupy the whole region at the crystallization front, thereby causing a monotonic increase in the crystal thickness to its upper part.

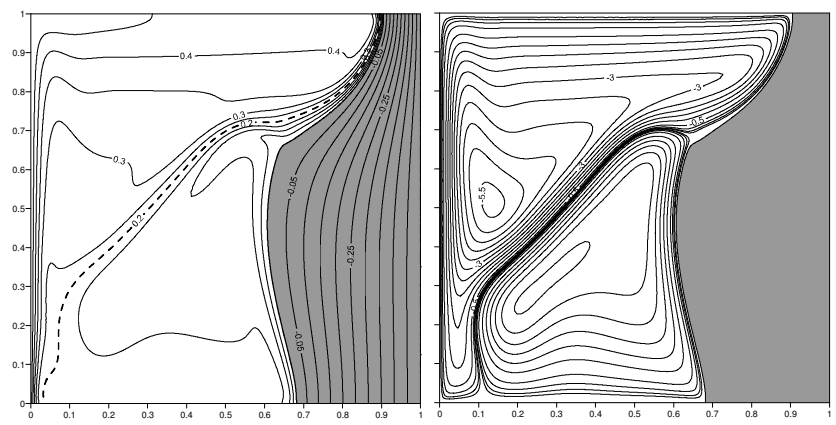

Fig. 2. Fields of isotherms and stream functions in steady state: height $=105 \mathrm{~mm}$, width $=105 \mathrm{~mm}$. Upper boundary with no-slip condition.

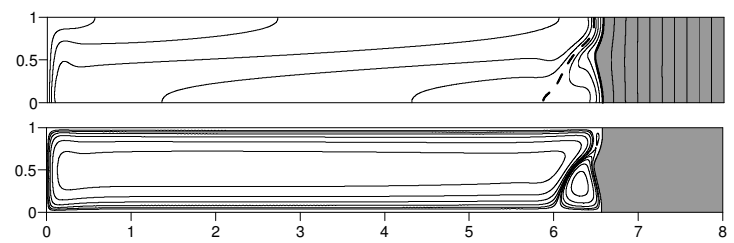

Fig. 3. Fields of isotherms and stream functions in steady state:height $=25 \mathrm{~mm}$, width $=200 \mathrm{~mm}$. Upper boundary with no-slip condition.

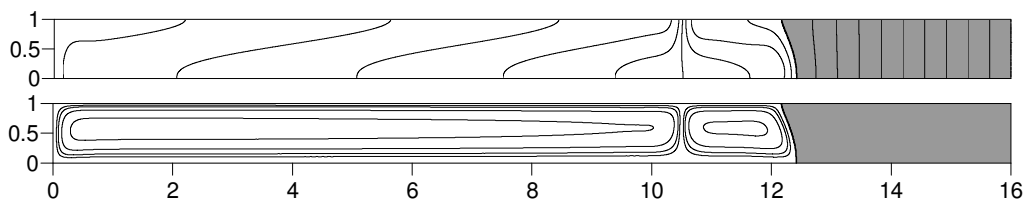

Fig. 4. Fields of isotherms and flow functions in steady state: height $=12.5 \mathrm{~mm}$, width $=200 \mathrm{~mm}$. Upper boundary with no-slip condition.

\section{Conclusions}

The shape of the crystallization front depends on the processes of conjugate convective heat transfer at the phase boundary. The influence of different boundary conditions on the upper boundary is studied. The calculations were carried out by the finite element method taking into account the latent heat of the phase transition. The obtained data can be useful in analyzing the growth of crystals by the method of horizontal directed crystallization of substances with an temperature inverse dependence of the melt density.

The author wishes to express his gratitude and thanks to Professor Vladimir S. Berdnikov for his supervision of this work.

This research has been executed within the limits of the project III.18.2.5. Number of the state registration AAAA-A17-117022850021-3. 


\section{References}

1. Muller, G., Crystal Growth from the Melt, (1988).

2. Berdnikov V.S., Zabrodin A.G., Markov V.A., Fluid Mech., Soviet Research, 15, no. 1. P.118-133, (1986).

3. Berdnikov, V.S., Gaponov, V.A., and Kovrizhnykh, L.S., J. Eng. Phys. Thermophys., 74, no. 4, p. 999- 1006, (2001).

4. Berdnikov V.S., Kislitsyn S.A., Mitin K.A., Bulletin of the Russian Academy of Sciences: Physics, 81, no 10, p. 1251-1256, (2017). 